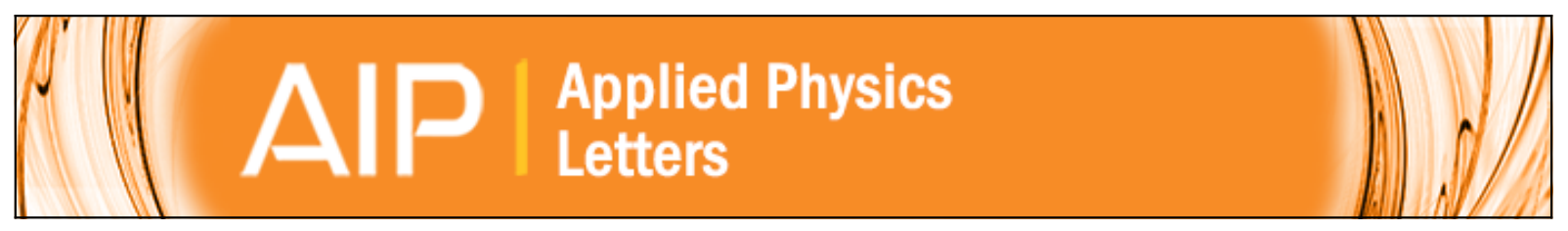

\title{
Electrostatic formation of liquid marbles and agglomerates
}

K. R. Liyanaarachchi, P. M. Ireland, G. B. Webber, and K. P. Galvin

Citation: Applied Physics Letters 103, 054105 (2013); doi: 10.1063/1.4817586

View online: http://dx.doi.org/10.1063/1.4817586

View Table of Contents: http://scitation.aip.org/content/aip/journal/apl/103/5?ver=pdfcov

Published by the AIP Publishing

\section{Articles you may be interested in}

Dynamic electrostatic force microscopy in liquid media

Appl. Phys. Lett. 101, 213108 (2012); 10.1063/1.4768164

The influence of electrostatic forces on the structure and dynamics of molecular ionic liquids

J. Chem. Phys. 128, 224503 (2008); 10.1063/1.2929848

Electrostatic Interactions and Complex Formation of $\mathrm{N}$-carboxyethylchitosan with Pectin

AIP Conf. Proc. 899, 817 (2007); 10.1063/1.2733558

Biofilm formation and local electrostatic force characteristics of Escherichia coli O157:H7 observed by electrostatic force microscopy

Appl. Phys. Lett. 90, 143901 (2007); 10.1063/1.2719030

A variation of electric wind experiment

Phys. Teach. 40, 315 (2002); 10.1119/1.1516391

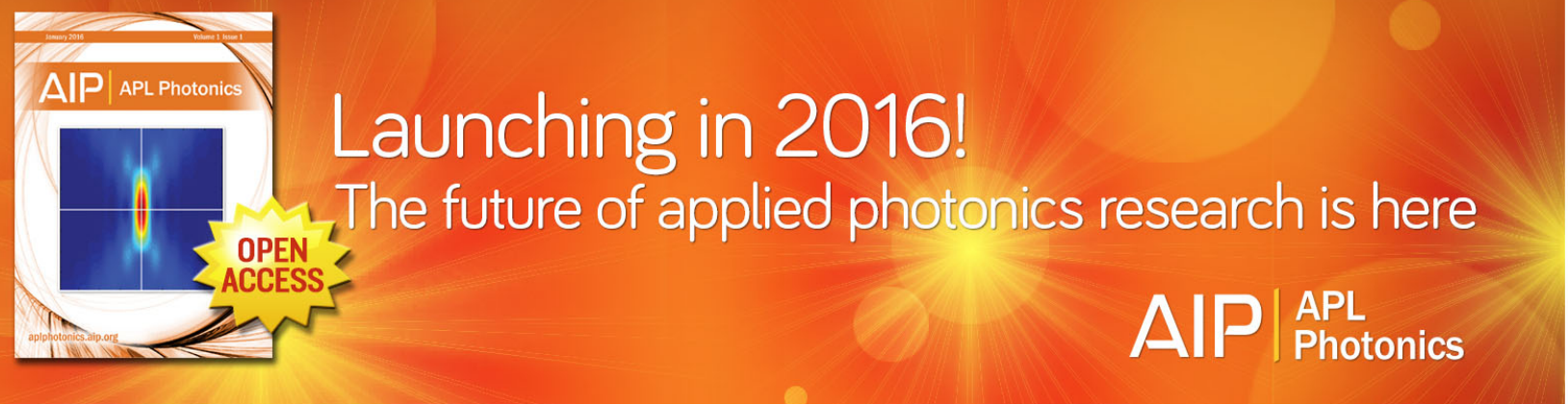




\title{
Electrostatic formation of liquid marbles and agglomerates
}

\author{
K. R. Liyanaarachchi, P. M. Ireland, ${ }^{\text {a) }}$ G. B. Webber, and K. P. Galvin \\ Centre for Advanced Particle Processing and Transport, School of Engineering, University of Newcastle, \\ Newcastle, Australia
}

(Received 21 May 2013; accepted 12 July 2013; published online 1 August 2013)

\begin{abstract}
We report observations of a sudden, explosive release of electrostatically charged $100 \mu \mathrm{m}$ glass beads from a particle bed. These cross an air gap of several millimeters, are engulfed by an approaching pendant water drop, and form a metastable spherical agglomerate on the bed surface. The stability transition of the particle bed is explained by promotion of internal friction by in-plane electrostatic stresses. The novel agglomerates formed this way resemble the "liquid marbles" formed by coating a drop with hydrophobic particles. Complex multi-layered agglomerates may also be produced by this method, with potential industrial, pharmaceutical, environmental, and biological applications. (C) 2013 AIP Publishing LLC. [http://dx.doi.org/10.1063/1.4817586]
\end{abstract}

It has always been difficult to harness, measure, and control electrostatic forces to perform precise tasks, including manipulation of small particles. ${ }^{1}$ This paper describes a curious electrostatic phenomenon, observed in our laboratory, which may assist in the formation of novel types of precisely structured particle agglomerates. A flat bed of micron-sized spherical ballotini particles was spread on a charged substrate, which was gradually raised towards a pendant water drop. $^{2}$ The substrate was wrapped in Polytetrafluoroethylene (PTFE) tape, which provided an ideal substrate for frictional charging, ${ }^{3}$ since it charges negatively in contact with most other materials. ${ }^{4}$ Particles were seen to intermittently jump across a gap of several millimeters to penetrate the surface of the drop. Eventually, a tipping point was reached, triggering a sudden "avalanche" of particles upward and into the drop. In around $300 \mathrm{~ms}$, the drop filled up like a "sack of marbles" before detaching. While the distance between the substrate and the drop was important in affecting particle lift, the substrate speed had relatively little effect on the onset of the particle "avalanche." Our findings suggest that an in-plane compressive stress develops in the upper layer of ballotini due to convergence of the electric field lines on the drop. This mechanism provides for a delayed release of the particles, which jump explosively to the drop instead of being gradually and incrementally stripped away as the separation decreases. Once detached, the particle-laden drop sits on top of the particle bed in a metastable state. This offers the intriguing possibility of forming water-based liquid marbles s, $^{5,6}$ with hydrophilic particles, or using combinations of hydrophilic and hydrophobic particles to form marbles with complex multilayered morphologies.

Figure 1 shows the process described above, with the image captured midway through the particle avalanche. We report here on our efforts to systematically reproduce, measure, control, and explain this complex process. In turn, we consider how it might be harnessed to develop new ways to manufacture functional multi-component particles with specific bulk and surface properties.

\footnotetext{
${ }^{\text {a) }}$ Author to whom correspondence should be addressed. Electronic mail: Peter.Ireland@newcastle.edu.au
}

The experimental system consisted of a bed of spherical ballotini particles nominally $100 \mu \mathrm{m}$ in diameter supported on a glass slide covered by PTFE tape. The PTFE tape was carefully applied around the glass slide, and rubbed 120 times, tribo-charging the surface. ${ }^{3,7}$ The experimenter used their index finger to rub the PTFE, while wearing a Nitrile glove ("Truecare Aloe," Ongard, Hornsby, Aust.). The glass ballotini was then added to the PTFE surface, ensuring no contact between the particles and the glass, resulting in the particles becoming charged by conduction. Any spillage of the particles across the PTFE and onto the glass slide led to loss of particle charge, and failure of the experiment.

A pendant drop of water was formed above the bed of particles via a stainless steel syringe, with the syringe connected to earth via the integrating circuit of an electrometer. The substrate was gradually driven upwards towards the drop using a mechanized micrometer stage at speeds ranging from 25 to $200 \mu \mathrm{m} \mathrm{s}^{-1}$. The drop profile was monitored using a DataPhysics OCA Tensiometer (Filderstadt, Germany).

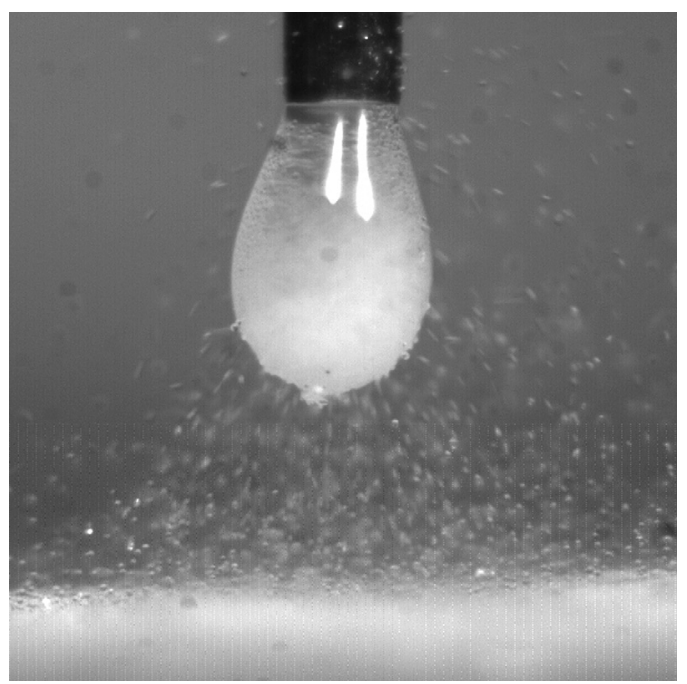

FIG. 1. Image showing particles of spherical ballotini being transferred to a pendant drop of water across an air gap. The total duration of this "avalanche" process is $\sim 300 \mathrm{~ms}$ (enhanced online) [URL: http://dx.doi.org/10.1063/ 1.4817586.1]. 


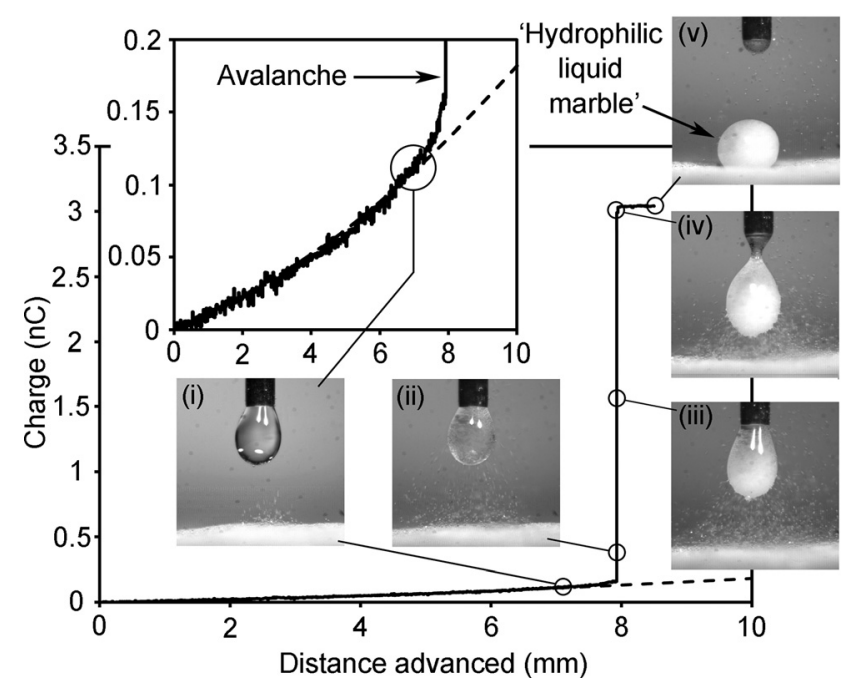

FIG. 2. Charge transferred to the drop when the stage is advanced at a steady velocity $\left(25 \mu \mathrm{m} \mathrm{s}^{-1}\right)$. The solid curve represents measured charge. The broken curve indicates the induction of image charge on the drop. The inset graph shows the same data prior to avalanche with an expanded vertical scale. The images correspond to the positions on the curve as indicated. (i) Initiation of particle transfer. (ii) Beginning of avalanche. (iii) Middle of avalanche. (iv) Detachment of drop, cessation of charge transfer-the drop is completely full of particles, like a "sack of marbles." (v) Metastable hydrophilic liquid marble remains on top of particle bed.

Figure 2 shows the cumulative charge transfer versus time, zero time corresponding to the beginning of the ascent of the substrate. When the micrometer stage was driven at a fixed speed, the total charge transfer recorded by the electrometer increased in a manner consistent with the induction of image charge on a spherical conductor approaching a negatively charged insulating plane. ${ }^{8}$ When the distance between the drop and the particle bed fell below a certain value, typically $2-4 \mathrm{~mm}$, particles began to jump to the drop under the influence of electrostatic forces. If the separation was reduced further, the most remarkable aspect of the process, an explosive release of particles from the substrate, was observed. Within a period of $300 \mathrm{~ms}$, the particles were swept upwards from the substrate and into the drop. The particles, which were fully engulfed by passing through the air-water interface, settled through the water toward the base of the drop. The additional weight continued to be supported by the surface tension and capillary force between the drop and the syringe tip. As a result, the drop "sagged" toward the substrate, decreasing the gap between the drop and the particles that remained on the substrate, further increasing the electric field strength and in turn inducing yet more particle transfer. Past a certain "tipping point," this positive feed-back mechanism overcame the restoring force associated with the surface tension, resulting in the detachment of the drop from the syringe. The explosive release of particles occurred within a very narrow range of separation distance between the drop and the substrate, independent of the rate of elevation in the micrometer stage. The amount of charge transferred during this avalanche also appears to have been independent of the stage velocity (Figure 3 ). In this and all other cases shown here, the drop fully detached from the needle well before making contact with the bed. Hence, the rapid increase in the measured value of the charge is not the result of direct conduction from the bed of silica particles to earth via a water "bridge."
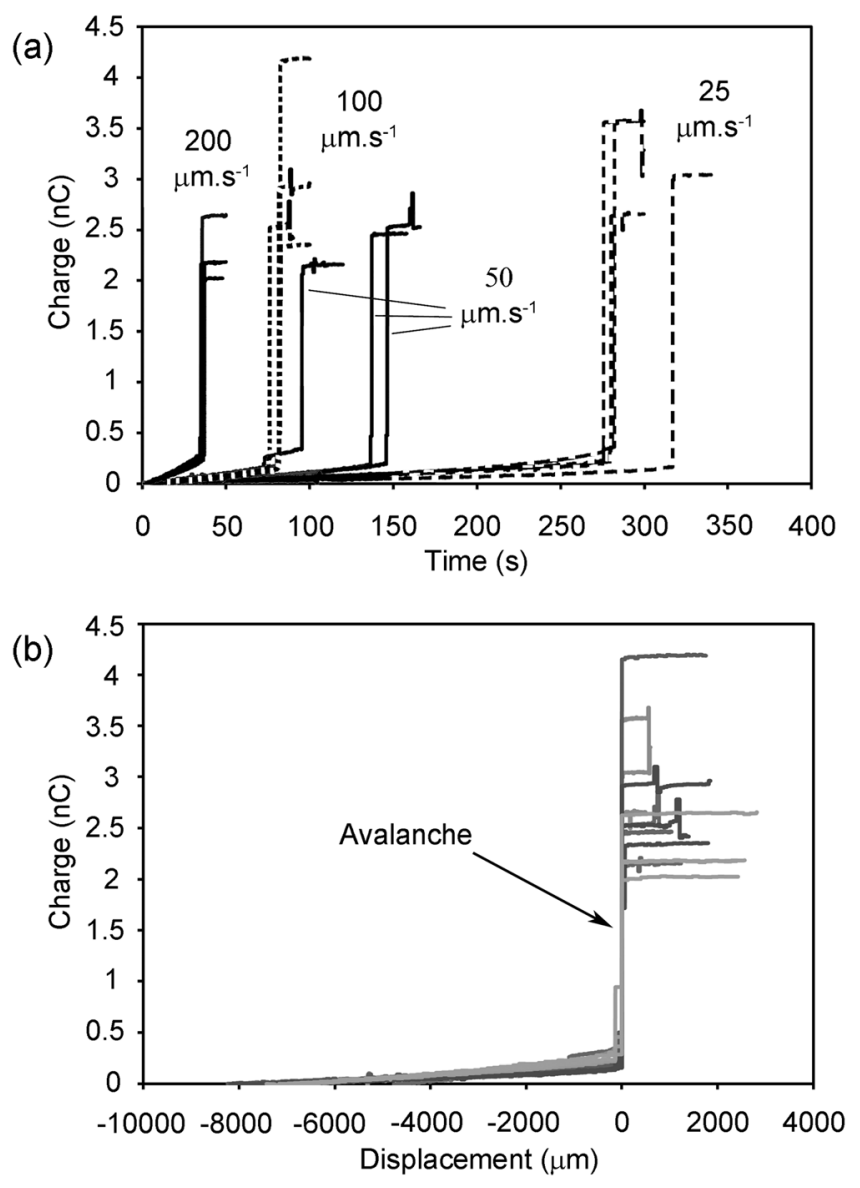

FIG. 3. Plots of recorded charge for constant-speed ascent experiments. (a) Plots of charge vs. time for an 8-fold increase in descent speed. Zero time corresponds to commencement of approach. (b) Plots of charge vs. displacement (i.e., substrate ascent time multiplied by ascent speed). These have been aligned using the main particle avalanche as a displacement benchmark, as indicated. Note that there is no discernible systematic relationship between the charge curve and the descent speed.

The rate of rise in the volume of the drop was measured and used to calculate the number rate addition of the ballotini spheres. By way of example, the volume increase in one experiment was found to be 1251 particles per second, while the electrometer showed an increase of $0.4 \mathrm{nC}$ over the same period. The ratio of these rates provided a measure of the charge, $q$, per particle, which was found to be $3.5 \times 10^{-13} \mathrm{C}$. By equating the force, $q E$, with the particle weight, $m g$, the electric field strength was estimated to be at least $21122 \mathrm{~N} \mathrm{C}^{-1}$.

It is clear that initiation of particle transfer is not simply a matter of the upward electrostatic force component exceeding the particle weight. High-speed video footage confirms that, at first, single isolated particles detach from the bed and accelerate upwards at $50-200 \mathrm{~m} \mathrm{~s}^{-2}$ while the surrounding particles remain in place. This implies the bed is behaving as a cohesive mass, undergoing structural rearrangement, and sometimes releasing individual particles. Bed cohesion can be explained in terms of inter-particle friction. The force on the particles above the substrate on either side of the axis of symmetry should have a radial inward component parallel to the substrate, in addition to the upward component, due to convergence of the electric field lines on the image charge on the drop (Figure 4). Both field components will increase 


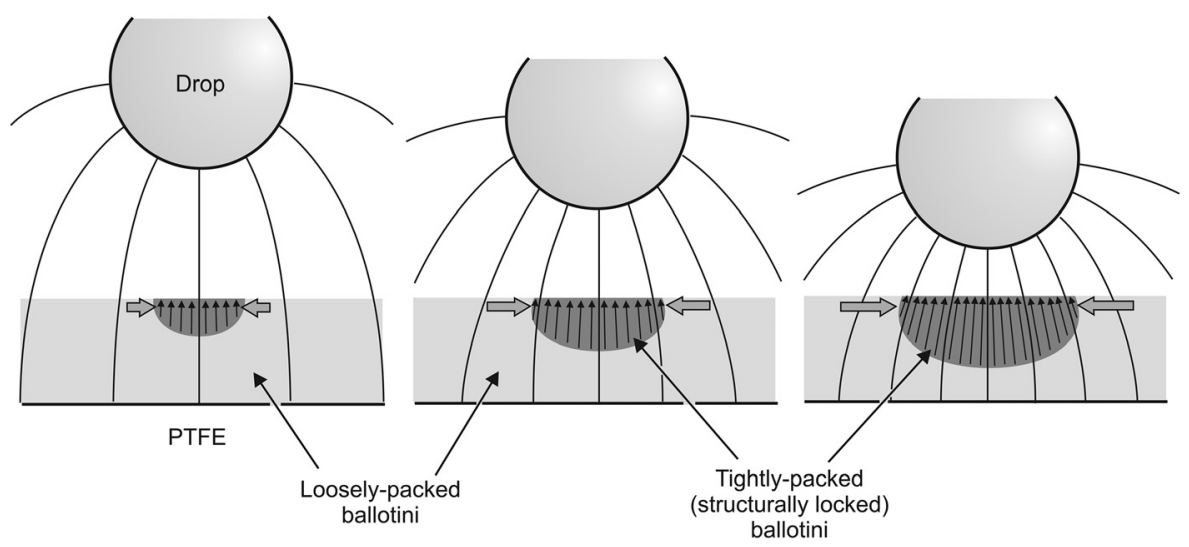

FIG. 4. Schematic of the proposed bed cohesion mechanism. The electric field lines converge on the drop, putting the particle bed immediately under the drop under a radial compressive stress in the plane of the bed surface. This prevents the particles being gradually stripped away as the gap closes, and instead favors discrete release events associated with bed rearrangements. as the drop-bed distance decreases. The radial inward component places the particles beneath the drop under a radial compressive stress. This stress increases inter-particle friction between the spheres and results in structural locking, opposing the vertical component of the force, and preventing vertical transport of the particles. As the stage is driven upward, the compressive stress increases, and the particles accordingly undergo a series of discrete stick-slip rearrangements. Some of these relieve the stress enough to release a quantity of particles. Eventually the system passes the "tipping point" described above, when the quantity of particles transferred is so great the surface tension force can no longer support the combined weight of the drop and particles, and the downwards electrostatic force.

Figure 5 shows two charge-time profiles where the stage was halted after the first particles were seen to jump to the drop, but before the final avalanche. In case (i), a series of particle transfer events occurred spontaneously over a period of $\sim 10 \mathrm{~s}$, eventually leading to an avalanche and detachment of the drop. These events were punctuated by periods of a number of seconds in which no transfer was observed. In case (ii), much larger spontaneous transfer events occurred approximately $20 \mathrm{~s}$ after the stage was halted, but this and other smaller transfers did not lead to an avalanche, possibly because the drop-bed separation was larger when particle transfer began. These experiments demonstrate that the system does not need to be continuously driven for particle transfer to occur. As the drop and bed are brought together, electrostatic potential energy is partially converted to stress in the particle bed. Even when the system ceases to be driven, this energy can remain stored. It is hypothesized that a relatively slow rearrangement process occurs in the apparently uneventful periods between particle releases, enabling each subsequent event. If particle release does not occur, the slow rearrangement process eventually leads to a genuinely stable state, as observed in case (ii).

The process was explored further using a screw micrometer instead of a motorized stage. Once the gap was small enough for particle transfer to begin, the stage was moved in rapid advances of a few tens of microns at a time. The accumulation in transferred particle charge, measured by the electrometer, reveals a strong correlation with the volume of the drop calculated using the tensiometer software, as shown in Figure 6. This is consistent with the observation that the particles are completely engulfed by the air-water interface, and add to the drop volume.
Hydrophobic particles can also be transferred to the drop. However, these are not engulfed, but instead form a packed domain at the surface of the drop, similar to the liquid marbles that are formed when a drop rolls across the bed. ${ }^{5}$ This packed layer appears to stabilize the drop surface and make it rigid. As a result, much less deformation is observed, which tends to suppress avalanches. In general, the system exhibits a more even and gradual particle transfer over a larger vertical distance than it does for hydrophilic particles.

Liquid marbles have been proposed for a wide range of applications over the last decade, including targeted drug delivery, cosmetics and cleaning agents and bio-reactors. ${ }^{6}$
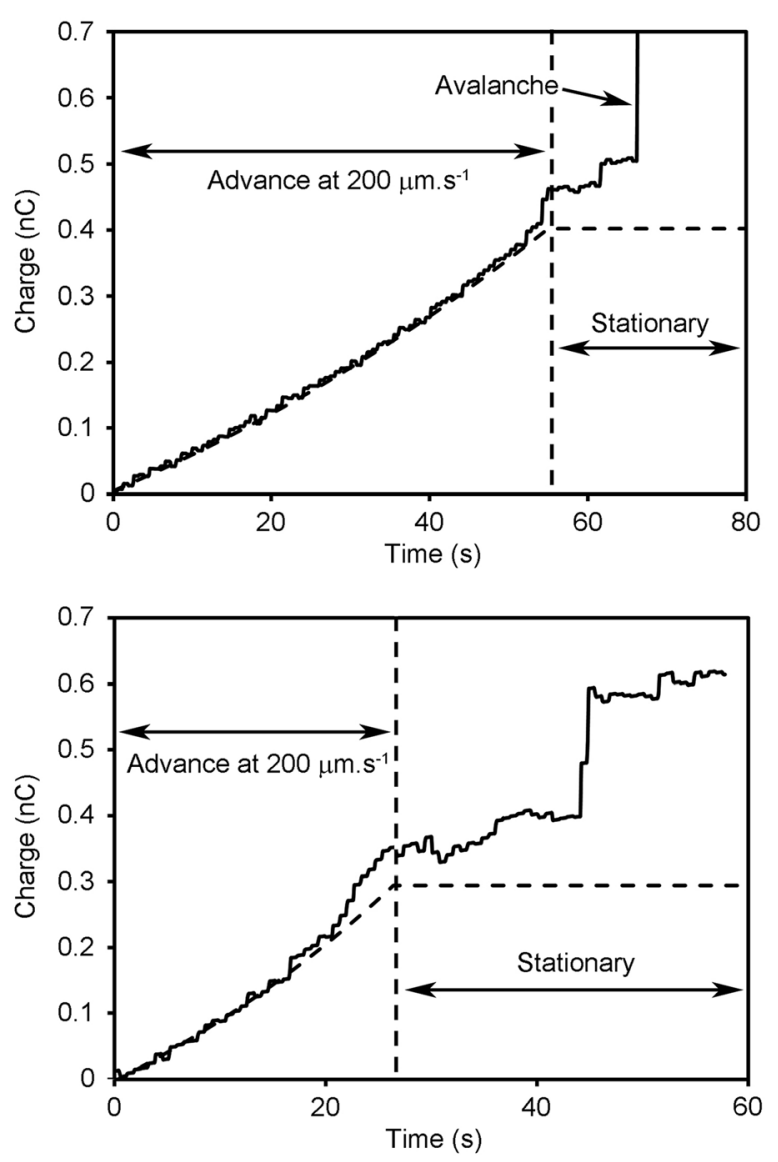

FIG. 5. Charge transferred to the drop when the stage is advanced at a steady velocity $\left(200 \mu \mathrm{m} \mathrm{s}^{-1}\right)$ then halted when particle transfer is observed. The solid curve indicates measured charge, and the broken curve indicates induction of charge on the drop. An avalanche occurred in the first case, but not in the second. Zero time represents commencement of the approach. 


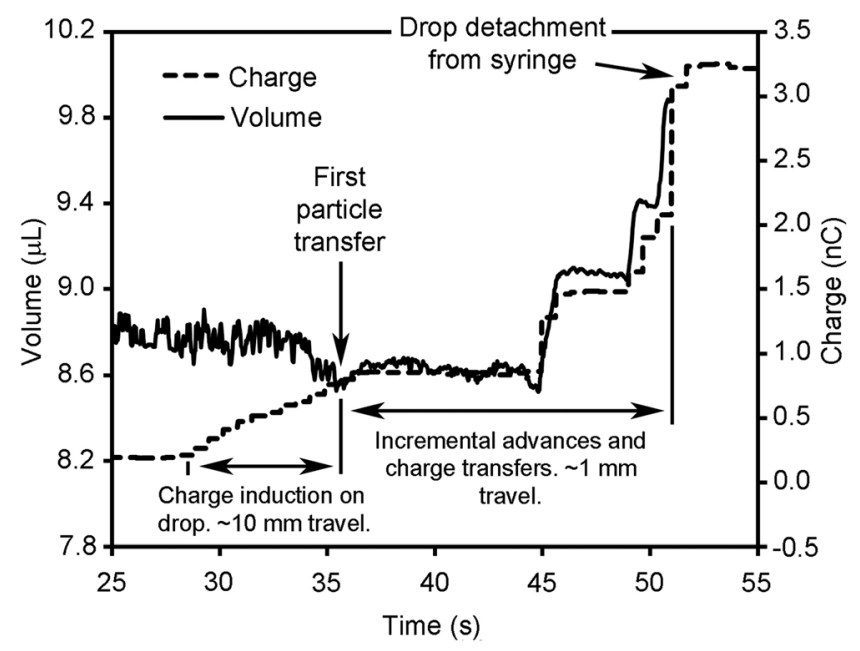

FIG. 6. Cumulative changes in the drop volume (solid curve), corresponding to cumulative changes in the charge transfer to the drop (broken curve). Subsequent to initial particle transfer, the volume and charge correspond closely. Commencement of the approach is at time $\sim 28 \mathrm{~s}$.

The phenomenon observed here may constitute an alternative way of forming them, with several attendant advantages. For example, marbles could be made with hydrophilic particles by this method (although the drop is filled, rather than merely coated, with particles). As clearly seen in Figure 2, an agglomerate of this type was left on top of the particle bed at the end of each experiment. Conversely, when a drop of liquid is placed on the surface of a hydrophilic particle bed, the liquid merely soaks in. While our agglomerates exhibited slow drainage of the binding liquid, and hence slumping, they could easily be stabilized in various ways.
For example, there is also the prospect of producing layered marbles, with a hydrophilic core and a stabilizing hydrophobic outer shell. Alternatively, the particles might undergo a form of sintering or freeze-drying. Since the formation of the marble does not require physical contact between the drop and the bed, it may also be possible to produce agglomerates that are far more symmetric than those formed by the more conventional method.

The authors acknowledge the support of Mark Mason in preparing the experimental arrangement. Dr. Ireland's participation was supported by the Australian Research Council via its Future Fellowship scheme (project number FT110100295). Support from the Australian Coal Association Research Program (ACARP) is gratefully acknowledged.

${ }^{1}$ J. A. Cross, Electrostatics: Principles, Problems and Applications (IOP Publishing, Bristol, 1987).

${ }^{2}$ C. E. Stauffer, "Measurement of surface tension by pendant drop technique," J. Phys. Chem. 69(6), 1933-1938 (1965).

${ }^{3}$ D. J. Lacks and R. M. Sankaran, "Contact electrification of insulating materials," J. Phys. D 44(45), 453001 (2011).

${ }^{4}$ A. R. Akande and J. Lowell, "Contact electrification of polymers by metals," J. Electrost. 16(2-3), 147-156 (1985).

${ }^{5}$ P. Aussillous and D. Quere, "Liquid marbles," Nature 411(6840), 924-927 (2001).

${ }^{6}$ G. McHale and M. I. Newton, "Liquid marbles: Principles and applications," Soft Matter 7(12), 5473-5481 (2011).

${ }^{7}$ A. G. Bailey, "The charging of insulator surfaces," J. Electrost. 51, 82-90 (2001); J. Lowell and A. C. Rose-Innes, "Contact electrification," Adv. Phys. 29(6), 947-1023 (1980).

${ }^{8}$ J. S. Berry and I. G. Higginbotham, "Electrostatic forces on a conducting sphere due to a charged, insulating plane," J. Phys. A 8(11), 1842-1851 (1975). 